TUMOUR IMMUNITY

\section{Plugging the gap}

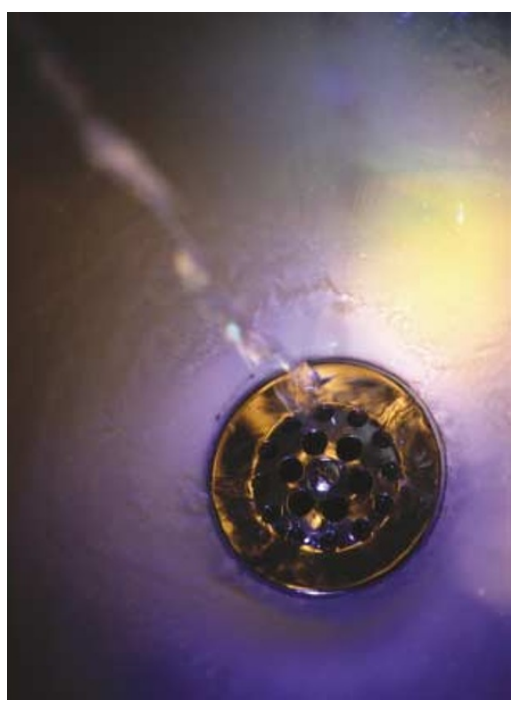

Most of the tumour antigens that have been identified are abberantly expressed self-molecules that are poorly immunogenic. This realization, together with the fact that normal individuals have $\mathrm{T}$ cells that react against self-antigens but are held in check by peripheral-tolerance mechanisms, has resulted in attempts to develop antitumour therapies that stimulate these antiself T cells and elicit antitumour responses. A new study in the Journal of Clinical Investigation shows that stimulating the homeostatic proliferation of a polyclonal population of adoptively transferred $\mathrm{T}$ cells - including those that are specific for tumour self-antigens in lymphopaenic animals can induce antitumour immunity.

Mice with lymphopaenia (induced by sublethal irradiation) and wild-type mice were challenged with a subcutaneous injection of melanoma cells, and tumour growth was assessed after 52 days. The lymphopaenic mice developed smaller tumours than wild-type mice. This antitumour effect was enhanced in a dose-dependent manner by the adoptive transfer of syngeneic lymph-node $\mathrm{T}$ cells before challenge with the tumour cells. In vitro testing of the homeostatically expanded $\mathrm{T}$ cells showed that they specifically recognized the melanoma tumour cells, but not unrelated colon carcinoma cells.

Next, the authors investigated whether T-cell clonal expansion per se was sufficient to induce the antitumour response or whether antigen presentation of tumour antigens was also important. The inhibition of tumour growth was more efficient in wild-type lymphopaenic mice transfused with $T$ cells and challenged with melanoma than in lymphotoxin- $\alpha$-deficient mice, which lack lymph nodes. When $T$ cells that cannot travel through lymph nodes (because they do not express $\beta 7$-integrin or L-selectin) were transferred, they were much less effective than normal $\mathrm{T}$ cells at eliciting the antitumour response. Together, these results show that the proliferating $\mathrm{T}$ cells need to be exposed to tumour antigens that are presented in the lymph nodes.

When mice that had rejected the melanoma cells were rechallenged with either melanoma or colon carcinoma cells, they again rejected the melanoma cells, but not the colon carcinoma cells, which indicates that the T-cell homeostatic proliferation had resulted in the development of

\title{
One small step for mankind...
}

The first oncogenic transcription factor, v-Jun, was discovered 15 years ago; shortly after this, its cellular counterpart, c-Jun, was also shown to be capable of transforming cultured cells. It has taken until now, however, to take that next great leap - to show that c-JUN is involved in human cancer. By investigating the DNA-binding activity of the AP1 transcription factor - a dimer that is composed of members of the JUN, FOS and ATF families - in a number of lymphoma cell lines, Stephan Mathas et al., reporting in The EMBO Journal, were able to show that AP1 was specifically activated in Hodgkin's lymphoma and anaplastic large-cell-lymphoma cell lines. The AP1 complex largely contained c-JUN, but another family member, JUNB, was also detectable in some of the cell lines; the increase in DNA-binding activity of AP1 correlated with an increase in protein levels of these components. This was confirmed when immunohistochemistry was performed on lymph-node biopsy samples, which were taken from patients with Hodgkin's disease.

AP1 is normally activated in response to extracellular signals, but is this also the case in Hodgkin's lymphoma? As expected, treating the lymphoma cell lines with phorbol esters activated the mitogenactivated protein kinase (MAPK) pathway and an AP1 complex; however, this was distinct from the one that is constitutively expressed in Hodgkin's disease - it was predominantly composed of c-FOS. c-JUN is also not phosphorylated in unstimulated cells, confirming that MAPK is not involved in its activation.

So, if JUN is activated independently of the normal extracellular signals, what does activate it? The remaining possibilities include increased transcription and decreased protein turnover. Pulse-chase experiments revealed that the half-life was unchanged, ruling out the latter option, but what of the former?

The c-JUN promoter is known to be autoregulated by AP1, which could therefore be responsible for the upregulation of c-JUN. To investigate this, a reporter construct containing either the wild-type or a mutated c-JUN promoter was transfected into a Hodgkin's lymphoma and a Burkitt's lymphoma cell line. The wildtype promoter was specifically activated in only the Hodgkin's lymphoma cells, indicating that AP1 is responsible for the activation of c-JUN.

The transcription factor NF- $\kappa B$ is also constitutively activated in Hodgkin's lymphoma cells, and transfecting these cells with an adenovirus that encoded an NF- $\mathrm{KB}$ repressor resulted in a decrease in the level of JUNB. Interestingly, this did not affect the activity of AP1, indicating that c-JUN is the more important AP1 component in these cells. So what effect does this increased AP1 activity have on Hodgkin's lymphoma cells? Its inhibition, using A-FOS, results in a decrease in cell division, which corresponds with a reduction in cyclin D2 expression; the proto-oncogene c-MET and the lymphocyte homing receptor CCR7 were also downregulated. Interestingly, the cellular response to inhibition of AP1 by A-FOS was different in anaplastic large-cell lymphoma cells - they underwent apoptosis, indicating that AP1 acts as a survival factor in these cells.

So, aberrant expression of JUN is involved in human cancer. This should strengthen the argument for the development of therapeutics that target this transcription factor.

Emma Greenwood

(2) References and links

ORIGINAL RESEARCH PAPER Mathas, S. et al. Aberrantly expressed c-Jun and JunB are a hallmark of Hodgkin lymphoma cells, stimulate proliferation and synergize with NF-кB. EMBO J. 21, 4104-4113 (2002) 